\title{
ESTUDO EXPERIMENTAL E SIMULAÇÃO DA DISPERSÃO DO TOLUENO NA FASE NÃO-SATURADA NO SOLO
}

\author{
E. A. S. CHIARAMONTE ${ }^{1}$ e R. M. KAUTZMANN ${ }^{3}$ \\ ${ }^{1}$ Universidade Federal do Pampa, Campus Bagé, Engenharia Química \\ ${ }^{2}$ Centro Universitário La Salle, Engenharia Ambiental \\ E-mail para contato: edsonchi@ portoweb.com.br
}

\begin{abstract}
RESUMO - A propagação dos compostos da gasolina em solo envolve os mecanismos de difusão, convecção e degradação. Esse trabalho mostra uma discussão dos mecanismos envolvidos na propagação e nas transferências de fases no solo. Nessa etapa é investigada a propagação do composto Tolueno em colunas de laboratório, simulando a zona não-saturada de um solo arenoso. 0 artigo mostra a metodologia experimental, para obtenção de valores quantitativos de concentração na coluna de laboratório. Ele também mostra os perfis de concentração obtidos para testes experimentais distintos e a discussão desses resultados numéricos obtidos.
\end{abstract}

\section{INTRODUÇÃO}

Os vazamentos de correntes de hidrocarbonetos (gasolina, diesel, querosene, nafta) para o solo têm chamado a atenção nos últimos anos, pois a propagação destes causam a contaminação do solo e podem atingir as águas subterrâneas, conforme Santos (1998), Cordazzo (2000) e Penner (2000). O transporte e a difusão de compostos hidrocarbonetos no solo envolve os fenômenos físicos como escoamento por gravidade, lixiviação, difusão mecânica e molecular. Os fenômenos de distribuição dos compostos entre as fases sólida, ar, água e óleo (adsorção na fase sólida, solubilidade na fase líquida, e transferência de massa para a fase vapor) e processos de degradação (química e biológica) também estão presentes. Nesta etapa da pesquisa investiga-se os fenômenos de dispersão na zona nãosaturada, Behrendt (2005) e Grathwohl et al. (2003). Portanto é importante o conhecimento da distribuição desses no solo e os mecanismos envolvidos na sua propagação: a difusão, a advecção e a degradação natural, conforme Nunes e Corseuil (2007). Também, são importantes as aplicações dos modelos matemáticos, que realizam a estimativa dessa propagação nos procedimentos de avaliação de impacto ambiental Fávero et al. (2007). Dados experimentais estão sendo obtidos numa coluna de solo em escala de laboratório. O composto Tolueno é usado como representativo da gasolina e valores quantitativos de concentração são obtidos ao longo da coluna. Este artigo contém os seguintes itens: a dispersão de compostos na zona não-saturada do solo, os materiais e os métodos para a obtenção de dados experimentais, os resultados experimentais e a conclusão desta etapa do trabalho. 


\section{A PROPAGAÇÃO dOS COMPOSTOS NA ZONA NÃO-SATURADA DO SOLO}

O transporte e a difusão de compostos hidrocarbonetos no solo envolve os fenômenos físicos de propagação como escoamento por gravidade, lixiviação, difusão mecânica e molecular, conforme Abriola e Pinder (1985). Os compostos também sofrem fenômenos de interação entre as fases e processos de degradação no solo. A região do solo em que o componente está se propagando também é um fator importante na modelagem da dispersão. O modelo deve levar em conta se é a fase nãosaturada ou saturada do solo. As primeiras camadas do solo são preenchidas por ar, umidade, e a fase orgânica nos seus vazios (zona não-saturada).

Considerando a não formação da fase de óleo na camada de solo e o mecanismo de propagação predominante, do composto orgânico, ser a difusão molecular no ar. A equação da difusão, que descreve a propagação unidimensional na zona não-saturada do solo, conforme Grathwohl et al. (2003) e Fetter (1998), é escrita na forma:

$$
\frac{\partial C_{i, a r}}{\partial t}=\frac{D_{E}}{\alpha} \frac{\partial^{2} C_{i, a r}}{\partial z^{2}}
$$

Onde t, z e $\mathrm{D}_{\mathrm{E}}$ são o tempo de liberação do composto, a distância na direção vertical e o coeficiente de difusão efetivo na fase ar do solo. O coeficiente de difusão é estimado pela relação de Millington e Quirk (1961):

$$
D_{E}=D_{i, a r} \frac{\left(\theta_{a r}\right)^{\frac{10}{3}}}{\theta^{2}}
$$

Onde $\mathrm{D}_{\mathrm{i}, \mathrm{ar}}$ e $\alpha$ e são o coeficiente de difusão do composto no ar e o parâmetro que leva em conta a distribuição do composto nas fases ar, água e material sólido do solo:

$$
\alpha=\theta_{a r}+\frac{\theta_{w}}{K_{h}}+\frac{K_{d} \rho_{b}}{K_{h}}
$$

Onde $K_{h}$ e $K_{d}$ são a constante da lei de Henry (adimensional) e o coeficiente de distribuição entre a fase sólida e a fase água. 
Nesse trabalho, o meio de propagação é limitado ao comprimento das colunas de laboratório, mostrado na Figura 2, e a solução matemática é aplicada a esse contorno limitado, conforme Ozisik (1993). São usadas duas soluções analíticas: a primeira com a condição de contorno de concentração fixa no topo da coluna (ponto de liberação; $\mathrm{z}=0$ ) e a segunda usa a condição de não existência de fluxo no topo e as duas aplicam a condição de fluxo de massa no fundo da coluna. As seguintes soluções matemáticas em série são obtidas:

A solução, para a condição de concentração fixa, é:

$$
C_{i, a r s}(z, t)=2 C_{i, o} \sum_{m=1}^{\infty} e^{-D_{E}^{2} B_{m} t} E_{m} F_{m}
$$

Onde as constantes $E_{m}$ e $F_{m}$ são:

$$
\begin{aligned}
E_{m} & =\frac{B_{m}^{2}+H_{2}^{2}}{L\left(B_{m}^{2}+H_{2}^{2}\right)+H_{2}} \\
F_{m} & =\frac{\cos \left(B_{m} L\right)-1}{B_{m}} \operatorname{sen}\left(B_{m} z\right)
\end{aligned}
$$

Onde as constantes $\mathrm{B}_{\mathrm{m}}$ são as raízes da equação:

$$
B_{m} \cot \left(B_{m} L\right)-H_{2}=0
$$

Onde $\mathrm{H}_{2}$ é a razão entre o coeficiente de transferência de massa no fundo da coluna e o coeficiente de difusão e L é o comprimento da coluna.

E, a solução para não-existência de fluxo é (Esta solução é uma liberação intermitente):

$$
C_{i, a r s}(z, t)=2 C_{i, o} \sum_{m=1}^{\infty} e^{-D_{E}^{2} A_{m} t} G_{m} H_{m}
$$


Onde as constantes Gm e Hm são:

$$
\begin{aligned}
G_{m} & =\frac{A_{m}^{2}+H_{2}^{2}}{A_{m} L\left(A_{m}^{2}+H_{2}^{2}\right)+H_{2} A_{m}} \\
H_{m} & =\operatorname{sen}\left(A_{m} L_{i}\right) \cos \left(A_{m} z\right)
\end{aligned}
$$

Onde as constantes Am são as raízes da equação:

$$
A_{m} \tan \left(A_{m} L\right)-H_{2}=0
$$

E, $\mathrm{L}_{\mathrm{i}}$ é o comprimento da liberação no ponto de emissão.

\section{METODOLOGIA EXPERIMENTAL}

\subsection{Elaboração das Soluções Padrões}

Uma quantidade pré-determinada do composto Tolueno é colocada num frasco de volume conhecido para a elaboração de misturas gasosas de concentrações conhecidas em ppm (parte por milhão) de massa.

\subsection{Procedimento de Liberação Experimental na Coluna de Solo}

Uma quantidade do composto Tolueno é liberada na coluna de solo. Depois de determinado período de tempo realiza-se a coleta em várias alturas da fração fluida do solo. Em seguida é determinada a concentração do composto no solo usando-se o método analítico de cromatografia gasosa.

O procedimento experimental consiste das seguintes etapas:

1) Preenchimento da coluna de bancada com areia fina (diâmetro da coluna $5 \mathrm{~cm}$ e comprimento de $1,5 \mathrm{~m})$

2) Liberação de uma quantidade definida de tolueno, representando um composto volátil da gasolina (desejado obter uma liberação instantânea);

3) Vedação da coluna no topo: 1. com gesso, visando reter a maior volatilização que ocorre quando exposto diretamente à atmosfera; 2. tampa de ferro soldada, para impedir qualquer perda para a atmosfera;

4) Coleta da amostra (fase fluida do solo) de contaminante em várias alturas da coluna. A amostra é composta da fase fluida do solo sem a presença da fase sólida. A coleta é realizada após um período de tempo determinado, utilizando uma seringa volumétrica.

5) Determinação da concentração quantitativa do contaminante na amostra. Utiliza-se o procedimento analítico de cromatografia gasosa. 


\section{RESULTADOS}

Na Figuras 1 é mostrado o resultado experimental do denominado teste 1. Ele é uma liberação de uma quantidade de $1 \mathrm{~mL}$ do composto Tolueno na coluna de solo. Essa foi vedada no topo com uma camada de gesso. A Figura 2 mostra o resultado do teste 2, que foi realizado nas mesmas condições do teste 1. A Figura 3 mostra o teste 3, onde a coluna está soldada no topo e foi emitido $0,5 \mathrm{~mL}$ de Tolueno. Na segunda figura foi colocada menos curvas para uma melhor visualização. Os gráficos mostram no eixo da abscissa distância do ponto de emissão (topo da coluna) contra concentração em ppm de massa. A primeira figura de cada teste mostra valores mais altos de concentração, pois apresenta os dias mais próximos da emissão. As Figuras 4 e 5 mostram resultados de simulação para os tempos de liberação de 1 e 2 dias para o teste 3. Foi usado um modelo unidimensional, que é uma combinação de liberações instantâneas. A solução analítica em série, para uma liberação instantânea, é dada pela relação (8).

\section{DISCUSSÕES DOS RESULTADOS}

Os resultados dos testes 1 e 2 mostram valores mais baixos de concentração para o composto Tolueno a partir do quarto dia no teste 1 e sexto dia no teste 2 (valores abaixo de $10000 \mathrm{ppm}$ ). Enquanto que, o teste 3, com a coluna soldada no topo, mantém mais altos valores de concentração até o décimo quinto dia. Os perfis de concentração também foram diferentes para a coluna vedada com o gesso e com a tampa soldada. Os perfis de concentrações parabólicos dos testes 1 e 2 mostram um escape do Tolueno no Topo da coluna, enquanto o teste 3 mostra um perfil exponencial, com a coluna isolada no topo. Os resultados das simulações mostram boa concordância e deverão ser realizadas análises estatísticas.
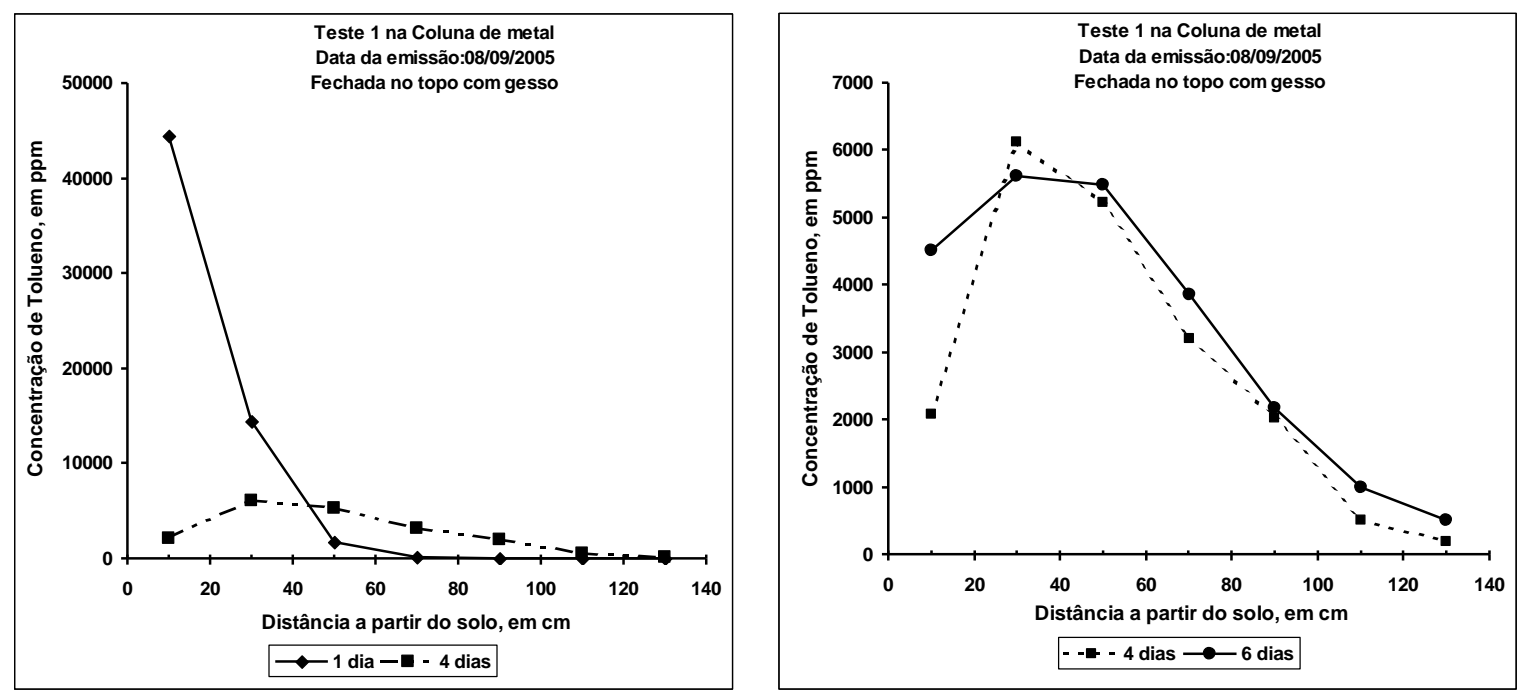

Figura 1. Liberação experimental no topo da coluna de solo (Teste 1). 

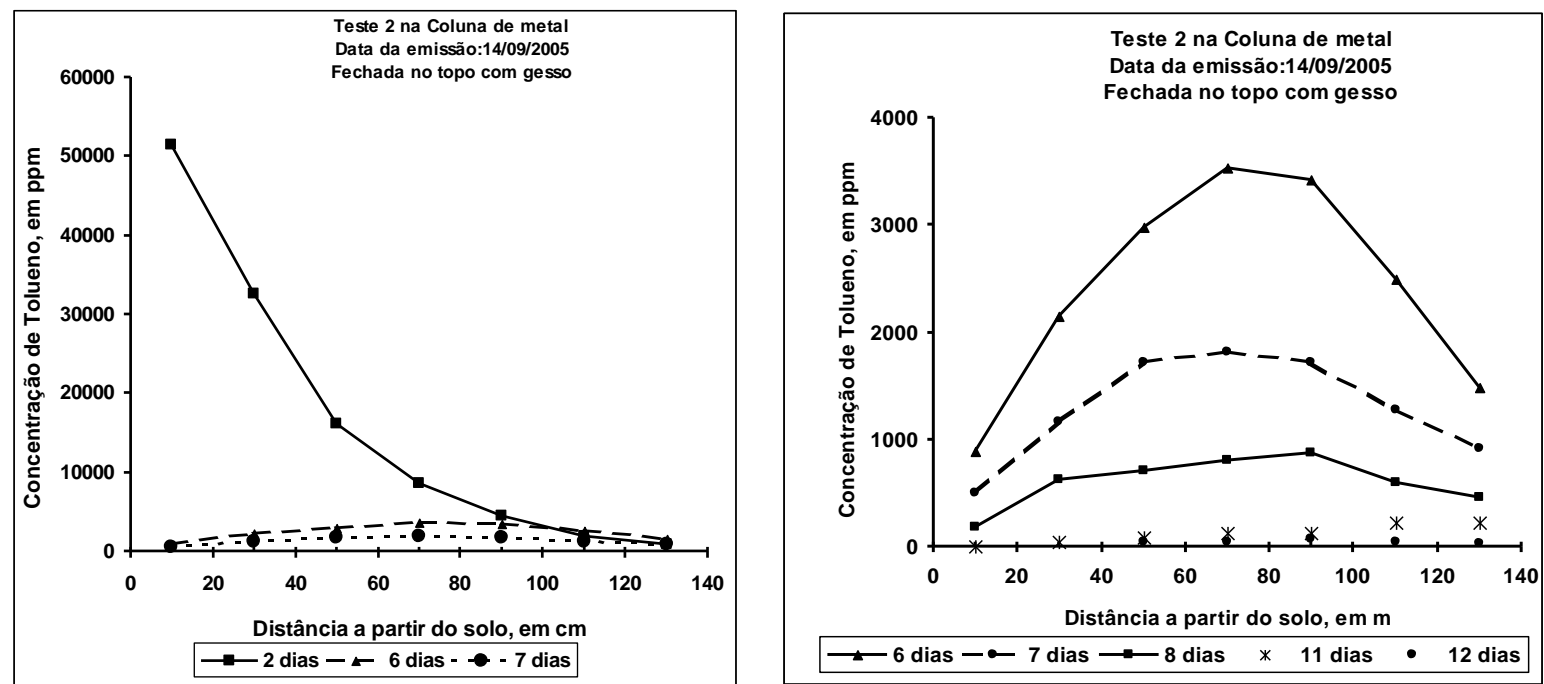

Figura 2. Liberação experimental no topo da coluna de solo (Teste 2).
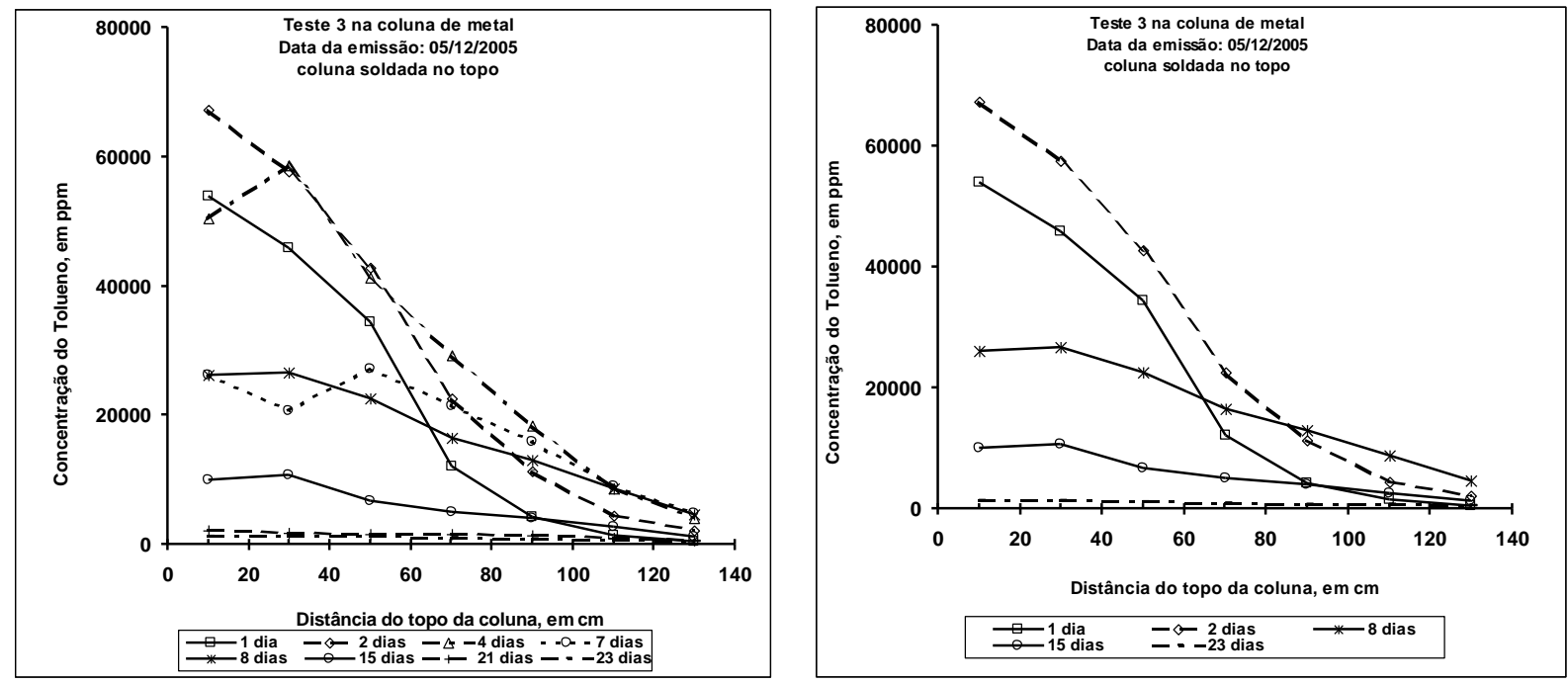

Figura 3. Liberação experimental com a coluna soldada no topo (Teste 3). 


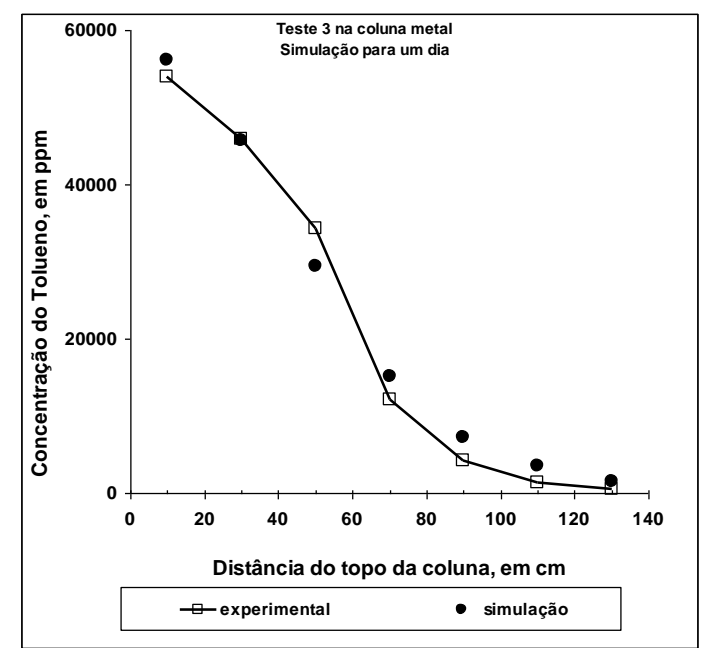

Figura 4. Simulação para o tempo de liberação de um dia para o teste 3.

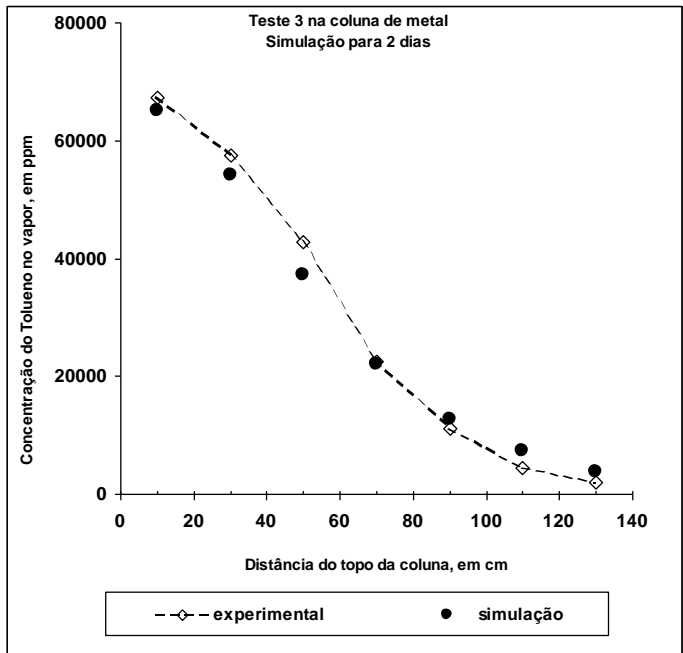

Figura 5. Simulação para o tempo de liberação de 2 dias para o teste 3.

\section{CONCLUSÕES}

Esta pesquisa investiga a propagação de compostos hidrocarbonetos em solo. A simulação experimental dessa propagação em colunas de laboratório com solo arenoso mostrou-se adequada, pois a técnica para a propagação do composto Tolueno obteve perfis de concentração que são semelhantes aos dos mecanismos da difusão na zona não-saturada do solo. $\mathrm{O}$ procedimento experimental apresentado mostrou-se adequado pelos valores de concentração obtidos e pela concordância com os resultados numéricos obtidos com o modelo físico de simulação. A dispersão de outros compostos como n-Heptano e Etanol deverão também ser investigados e também a propagação dos compostos em solo saturado de umidade são as próximas etapas da pesquisa. 


\section{REFERÊNCIAS}

ABRIOLA, L. M E PINDER, G. F. A Multiphase Approach to the Modeling of Porous Media Contamination by Organic Compounds, 1, Equation Development. Water Resources Res., v. 21, p. 11-18, 1985.

BEHRENDT, H. SNAPS/SOIL - water, heat, material transport in soil, 1996. Disponível: http://www.gsf.de/UFIS/ufis/modell17/modell.html, 2005.

CORDAZZO, J. Modelagem e Simulação Numérica do Derramamento de Gasolina Acrescida de Álcool em Águas Subterrâneas, Dissertação de Mestrado, UFSC, 2000.

FÁVERO, J, L., MUNIZ, A. R. E SANTOS, R. P. B. Análise Teórico-Experimental da Dispersão de Poluentes Líquidos em Solos Eng. Sanit. Ambient., v.12, n.4, p. 410, Rio de Janeiro, 2007.

FETTER, C. W. J. Contaminant Hydrogeology, New York, Macmillan Publishing Co., 2nd Edition, 1998.

GRATHWOHL, P.; WANG, G.; BREDERODE, S.; RECKHORN, F. Volatile Organic Compounds Volatilization from Multicomponent Organic Liquids and Diffusion in Unsatured Porous Media, Vadose Zone Journal, v. 2, p. 602-701, 2003.

JURY, W. A.; GARDNER, W. R.; GARDNER, W. H. Soil Physics, 6th Edition (Wiley), 2005.

MILLINGTON, R. J. E QUIRK, J. M. Permeability of porous solids, p. 97-106. 7th International Congress of Soil Science. SSSA, Madison, 1960.

NUNES, C. N. E CORSEUIL, H. X. (2007) Importância do etanol na atenuação natural de águas subterrâneas impactadas por gasolina, Eng. Sanit. Ambient., v.12, n.3, Rio de Janeiro.

SANTOS, A. H. P. Simulação Composicional para Transporte de Hidrocarbonetos em Aqüíferos, Dissertação de Mestrado, Unicamp, 1998.

OZISIK, M. N. Heat Conduction, John Wiley Professional, 2a Edição, 1993.

PENNER, G. C. Estudo Laboratoriais da Contaminação do Solo com Gasolina com o Uso de um Detetor de Fotoionização, Dissertação de Mestrado, Universidade de São Paulo, São Carlos, 2000. 